\title{
A Low-Power Current-Reuse LNA for 3D Ultrasound Beamformers
}

\author{
Yohei NAKAMURA $^{\dagger \text { a) }}$, Shinya KAJIYAMA ${ }^{\dagger}$, Members, Yutaka IGARASHI ${ }^{\dagger \dagger}$, Nonmember, $^{\prime}$ Takashi OSHIMA ${ }^{\dagger}$, \\ and Taizo YAMAWAKI ${ }^{\dagger}$, Members
}

\begin{abstract}
SUMMARY 3D ultrasound imagers require low-noise amplifier (LNA) with much lower power consumption and smaller chip area than conventional 2D imagers because of the huge amount of transducer channels. This paper presents a low-power small-size LNA with a novel currentreuse circuitry for 3D ultrasound imaging systems. The proposed LNA is composed of a differential common source amplifier and a source-follower driver which share the current without using inductors. The LNA was fabricated in a $0.18-\mu \mathrm{m}$ CMOS process with only $0.0056 \mathrm{~mm}^{2}$. The measured results show a gain of $21 \mathrm{~dB}$ and a bandwidth of $9 \mathrm{MHz}$. The proposed LNA achieves an average noise density of $11.3 \mathrm{nV} / \sqrt{ } \mathrm{Hz}$, and the $2 \mathrm{nd}$ harmonic distortion below $-40 \mathrm{dBc}$ with $0.1-\mathrm{Vpp}$ input. The supply current is $85 \mu \mathrm{A}$ with a $1.8-\mathrm{V}$ power supply, which is competitive with conventional LNAs by finer CMOS process.

key words: amplifier, current-reuse, driver, ultrasound, LNA
\end{abstract}

\section{Introduction}

Ultrasound imaging systems are widely used for medical diagnosis. A 1D array probe is commonly used to obtain 2D images, but 3D imaging systems with $2 \mathrm{D}$ array probes have been used recently to minimize examination time and to make it easier to find a target [1]-[3]. In the conventional 1D probe, a received ultrasound signal is converted into an electrical signal at a transducer array in the handheld probe and usually transmitted to a main unit that has a beamfocusing function as shown in Fig. 1(a). However, for the 2D array probe, this function must be integrated in the probe as shown in Fig. 1(b) to avoid a too heavy transceiver cable to the main unit necessary for accommodating massive channels of the ultrasound transmitter and receiver. Fig. 2 shows an architecture of an ultrasound beamformer IC. The frontend transducer is connected to a low-noise amplifier (LNA) through a transmitter (TX) and receiver (RX) switch (TRSW) [4]. A received ultrasound signal is converted into an electrical signal, amplified in the LNA stage, and sent to a variable delay stage and a subsequent adder stage. The variable delay stage decides the focus point of the ultrasound image. In such a $2 \mathrm{D}$ matrix probe, the power and area budget given to individual channels is significantly limited be-

Manuscript received June 1, 2020.

Manuscript revised September 20, 2020.

The author is with the Center for Technology Innovation Electronics, Research and Development Group, Hitachi, Ltd., Tokyo, 185-8601 Japan.

The author is with the Center for Technology Innovation Production Engineering, Research and Development Group, Hitachi, Ltd., Yokohama-shi, 244-0817 Japan.

a)E-mail: yohei.nakamura.sj@hitachi.com DOI: $10.1587 /$ transfun.2020GCP0011

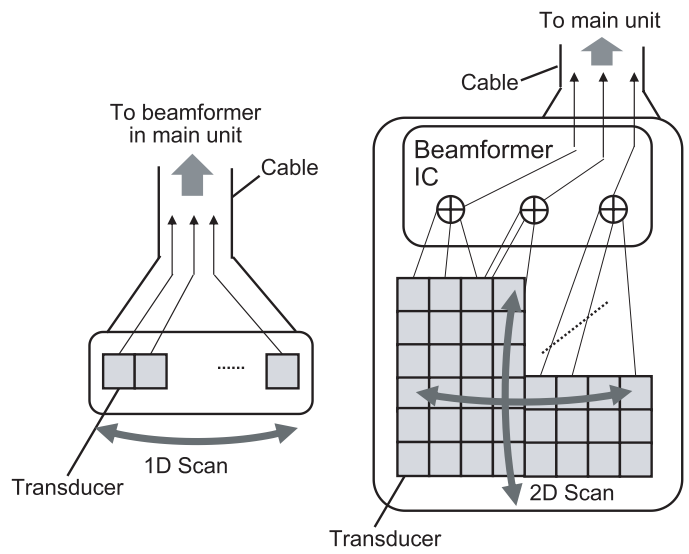

Fig. 1 Probes for ultrasound imagers. (a) 1D array for 2D imager. (b) $2 \mathrm{D}$ array for $3 \mathrm{D}$ imager.

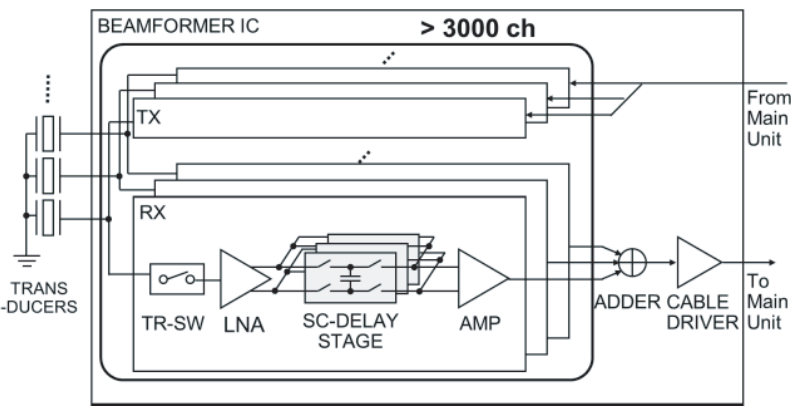

Fig. 2 Architecture of ultrasound beamformer IC

cause 1,000 to 10,000 channels must be integrated in a small hand-held probe. For example, to achieve a power consumption of $1 \mathrm{~W}$ with 3,000 channels, assuming the TX and RX budget to be 1:1, the power consumption of an individual receiver channel must be $<150 \mu \mathrm{W}$, which is further divided into each of the stages that constitute the RX channel. Nevertheless, additional noise at the LNA must be sufficiently small so that very weak signal from deep structures in the human body can be captured. Another crucial requirement for the LNA is that it must drive the delay stage, which is composed of a high-speed parallel switched capacitor (SC) circuit. Furthermore, 2nd harmonic distortion (2HD) of the LNA must also be suppressed to a specified level because 2nd harmonic ultrasound signals are used in some of the imaging modes used for diagnostic procedures.

In some low-power LNAs used for RF applications, 
the current-reuse technique is known as an attractive solution that reduces power consumption [5]-[7]. The currentreuse architecture of RF-LNAs usually uses an inductor element to isolate between the functional stages. However, the frequency band of medical ultrasound imagers is usually around $1-10 \mathrm{MHz}$, which is much lower than that of RF-LNAs. The inductor size becomes prohibitively large to be applied for this frequency band. Some other current-reuse architectures for LNA were also proposed to increase transconductance or reduce power consumption of several receiver channels without using inductors [8]-[10]. However, a current-reuse architecture optimized for driving switched capacitor circuit in 2D array probe has not been proposed.

In this paper, a low-power small-size current-reuse LNA without an inductor is proposed and verified by the prototype measurement for ultrasound beamformer ICs. The proposed current-reuse circuitry enables independent optimization of both a high gain at the amplifier stage and low output impedance at the driver stage with halved power consumption. A differential architecture is employed to suppress 2 HD. In addition, both amplifier and driver stages are NMOS-based for even better power and area efficiency. This paper is organized as follows. In Sect. 2, the architecture and operating principle of the circuit are described. The stability is analyzed in Sect. 3. The circuit is verified by measurement in Sect. 4, and a comparison with other low-power LNAs for ultrasound systems is shown. Section 5 concludes the paper.

\section{Proposed Architecture of LNA}

Figure 3(a) shows a conventional current-reuse RF-LNA with a driver stage [5]. This LNA uses two inductors as a load without DC drop and the structure is composed of five-stacked functional elements as described in right-side of Fig. 3(a). The differential architecture is suitable for the cancellation of $2 \mathrm{HD}$ required by the ultrasound system. However, inductors for load functions are not suitable for the frequency range of the ultrasound system as mentioned previously. On the other hand, the voltage headroom is sacrificed if inductors are replaced by MOS transistors. Thus, Fig. 3(b) shows a proposed inductor-less current-reuse LNA with a driver stage. A differential common-source (CS) amplifier stage is located on top of a source-follower (SF) driver stage in order to reuse the bias current. The common drain nodes of the transistors in the driver stage and the common source nodes of the transistors in the amplifier stage are connected directly as an AC ground without using an inductor. This structure also reduces the number of stacked elements from five to four, which provides larger voltage headroom while all loads are implemented with MOS transistors. Another significant feature is that NMOS transistors are used for input of the source follower by flipping the PMOS counterpart in [5]. Because a required size of NMOS transistor is smaller than PMOS to obtain same transconductance, the load capacitances of the amplifier stage are reduced. Therefore, the further power saving is attained to achieve the re-

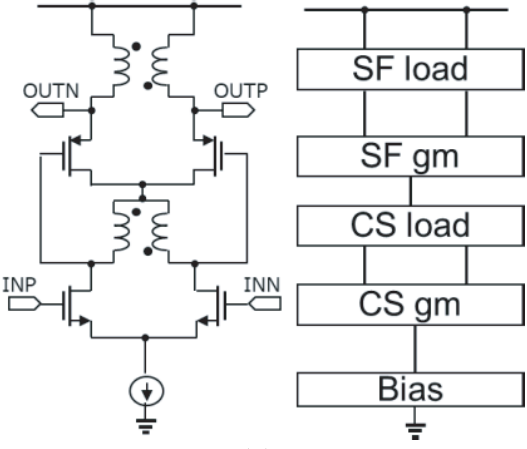

(a)

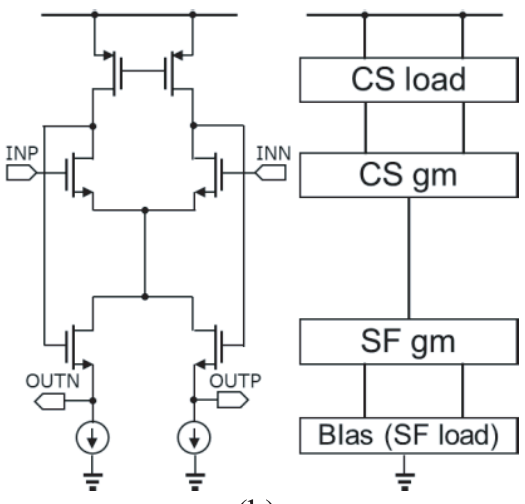

(b)

Fig. 3 Architecture of conventional [5] and proposed current-reuse LNA (a) Conventional LNA. (b) Proposed LNA.

quired bandwidth as well as the area saving.

In Fig. 4, a full schematic of the proposed LNA is shown. For the amplifier stage, the driver stage is seen as a high-impedance current source because the current flow through the junction node $(\mathrm{P})$ is kept constant set by the bottom transistors M7 and M8. The impedance of the current source is determined by the channel-length modulation effect of M7 and M8. When the input signal $\left(v_{I N}\right)$ is singleended (as required by ultrasound imagers) and the gate voltage of M2 is a fixed DC bias as shown in Fig. 4, the voltage of junction node $\mathrm{P}\left(\mathrm{v}_{\mathrm{P}}\right)$ follows the same polarity as $\mathrm{v}_{\mathrm{IN}}$ because the common-mode input component appears at this node. The drain current of M2 is also generated by the swing of $\mathrm{v}_{\mathrm{P}}$. This results in the positive output of the $1 \mathrm{st}$ stage $\left(\mathrm{v}_{\mathrm{O} 1 \mathrm{P}}\right)$ having the same polarity as $\mathrm{v}_{\mathrm{IN}}$ and the negative output having the opposite polarity. At the sourcefollower stage, the voltage of $\mathrm{V}_{\mathrm{O} 1 \mathrm{P}}$ and $\mathrm{V}_{\mathrm{O} 1 \mathrm{~N}}$ is shifted by $\left(\mathrm{V}_{\mathrm{TH}}+\mathrm{V}_{\mathrm{OV}}\right)$ with M3 and M4. Here, $\mathrm{V}_{\mathrm{TH}}$ and $\mathrm{V}_{\mathrm{OV}}$ are the threshold voltage and over-drive voltage of the MOS transistors, respectively. The body terminals of M3 and M4 are connected to their source terminals to avoid the body effect. One of the important points of this structure is that it enables a high gain with a low output impedance because the maximum gain is determined by the output impedance of the 1st amplifier stage, and a low output impedance is achieved in the driver stage.

The proposed stacked architecture needs to be care- 


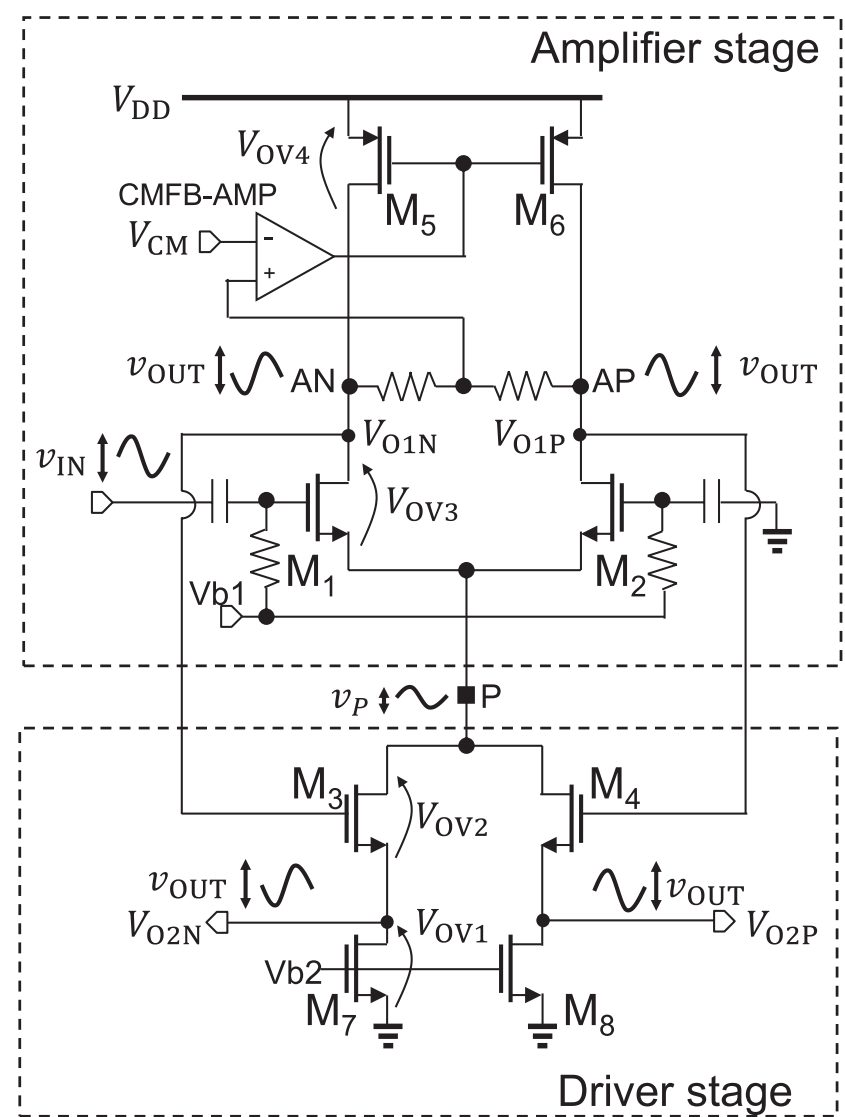

Fig. 4 Schematic of proposed LNA circuit.

fully designed in consideration of the bias point and voltage swing at each node. A simple guideline for the design is given as follows. As previously described, the polarity of the voltage swing at the drain and source is the same for M2 and M4 but opposite for M1 and M3. Therefore, the constraint for operating the transistors in the saturation region for M1 and M3 is more stringent than that for M2 and M4. The condition for operating in the saturation region for M1, M3, and M5 is described as (1) and (2).

$$
\begin{aligned}
& V_{\mathrm{OV} 1}+v_{\mathrm{OUT}} \\
& +V_{\mathrm{OV} 2}+v_{\mathrm{P}}+V_{\mathrm{OV} 3}+v_{\mathrm{OUT}}+V_{\mathrm{OV} 4}<V_{\mathrm{DD}} \\
& v_{\mathrm{P}}=\frac{v_{\mathrm{IN}}}{2}, \quad v_{\mathrm{OUT}}=\frac{G}{2} v_{\mathrm{IN}}
\end{aligned}
$$

Here, $\mathrm{V}_{\mathrm{OVX}}(\mathrm{X}=1 \sim 4)$ is over-drive voltage of each transistor as denoted in Fig. 4 and $\mathrm{v}_{\mathrm{OUT}}, \mathrm{v}_{\mathrm{P}}$, and $\mathrm{v}_{\mathrm{IN}}$ are voltage swing at the output of driver, node $\mathrm{P}$ and input of amplifier stage respectively. $\mathrm{G}$ is the differential voltage gain of the amplifier. In (1), the voltage gain of the source follower (M3, M4) was approximately regarded as one, which is reasonable when the body terminal is connected to the source terminal. (1) and (2) result in the following Eq. (3).

$$
V_{\mathrm{OV} 1}+\left(G+\frac{1}{2}\right) v_{I N}+V_{\mathrm{OV} 2}+V_{\mathrm{OV} 3}+V_{\mathrm{OV} 4}<V_{\mathrm{DD}}
$$

10 , (3) results in $\mathrm{v}_{\mathrm{IN}}<100 \mathrm{mV}$, giving upper limit of the input range. We also need to consider the voltage shift by the source follower as follows.

$$
V_{\mathrm{O} 2}=V_{\mathrm{O} 1}-V_{\mathrm{TH}}-V_{\mathrm{OV} 2}
$$

Due to this relation, the 1 st-stage common-mode level $\left(V_{\mathrm{O} 1}\right)$ is determined by the output common-mode level $\left(\mathrm{V}_{\mathrm{O} 2}\right)$. Also, the voltage swing at junction node $\mathrm{P}$ and the output node is sandwiched by $\mathrm{V}_{\mathrm{O} 1}$ and $\mathrm{V}_{\mathrm{O} 2}$, which gives Eq. (5) and hence (6) by combining with (4).

$$
\begin{aligned}
& V_{\mathrm{O} 2}+\frac{v_{\mathrm{OUT}}}{2}+V_{\mathrm{OV} 2}+v_{\mathrm{P}}<V_{\mathrm{O} 1}-\frac{v_{\mathrm{OUT}}}{2}-V_{\mathrm{OV} 3} \\
& v_{\mathrm{OUT}}<\frac{V_{\mathrm{TH}}-V_{\mathrm{OV} 3}}{1+1 / \mathrm{G}} \approx V_{\mathrm{TH}}-V_{\mathrm{OV} 3}
\end{aligned}
$$

The Eq. (6) means that the maximum output swing is determined by the threshold voltage of M3 and M4. Thus, to achieve the maximum swing of $\mathrm{v}_{\text {OUT }}=0.5 \mathrm{~V}$, the threshold voltage needs to be equal to or larger than $0.7 \mathrm{~V}$. From these analyses, when $\mathrm{V}_{\mathrm{OVX}}=0.2 \mathrm{~V}$ and $\mathrm{V}_{\mathrm{TH}}=0.7 \mathrm{~V}$, the appropriate bias voltage can be decided to be about $\mathrm{V}_{\mathrm{O} 2}=0.45 \mathrm{~V}$ and $\mathrm{V}_{\mathrm{O} 1}=1.35 \mathrm{~V}$. Here, $\mathrm{V}_{\mathrm{O} 1}$ is controlled to be the same voltage as common-mode bias $\mathrm{V}_{\mathrm{CM}}$ by a common-modefeedback amplifier (CMFB-AMP) and load PMOS transistors (M5, M6) as shown in Fig. 4. Once $\mathrm{V}_{\mathrm{O} 1}$ is set by $\mathrm{V}_{\mathrm{CM}}$, $\mathrm{V}_{\mathrm{O} 2}$ is also biased automatically with the relationships in accordance with Eq. (4).

Next, the effects of process, voltage and temperature variations are discussed. The proposed LNA is affected by variation of the threshold voltage of MOS transistors (due to process or temperature variations) more significantly than by variation of the over-drive voltage $\left(\mathrm{V}_{\mathrm{OVX}}\right)$, as long as the bias circuit is designed to keep the current constant against the variations. As shown in (6), the maximum output swing is determined by the threshold voltage and becomes smaller with the lower threshold voltage. Therefore, the output range and hence the input range of the proposed LNA need to be set by considering the minimum threshold voltage under the variations. In addition, when the power supply voltage decreases, the input range can be reduced based on (3).

It should also be noted that the source follower serves not only as a driver but also as a level shifter. The delay stage includes parallel samplers which are composed of MOS switches and capacitors as shown in Fig. 5(a). A bandwidth of the samplers is determined by an output impedance ( $\mathrm{R}_{\mathrm{OUT}}$ ) of the LNA, an on-resistance of the sampling switch $\left(\mathrm{R}_{\mathrm{SW}}\right)$ and a capacitance of the sampling capacitor in each sampler. The low-level voltage $\left(\mathrm{V}_{\mathrm{O} 2}\right)$ brought by the levelshift function of the source follower makes it possible to use NMOS switches instead of CMOS switches because the on-resistance of NMOS is lower when the signal stays in low level as shown in Fig. 5(b). This is additional advantage for reducing the area of the ultrasound beamformer IC since there are many sampling switches in the delay stage.

When applying $\mathrm{V}_{\mathrm{DD}}=1.8 \mathrm{~V}, \mathrm{~V}_{\mathrm{OVX}}=0.2 \mathrm{~V}$, and $\mathrm{G}=$ 


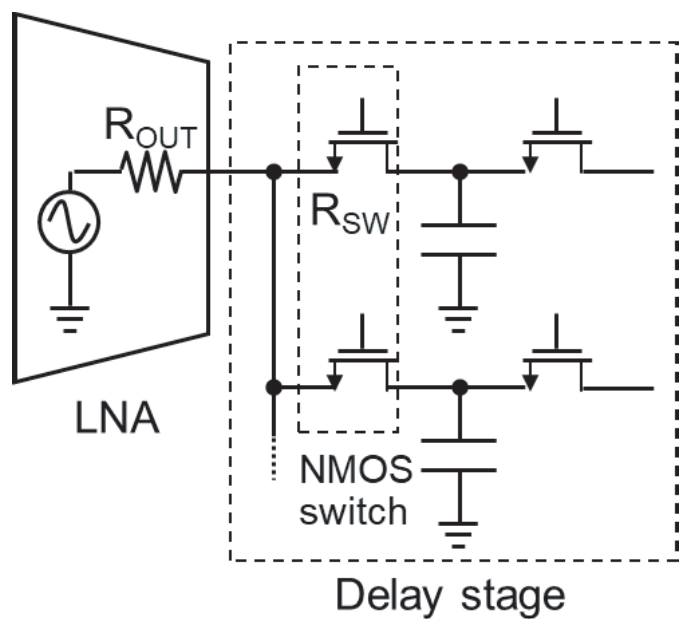

(a)

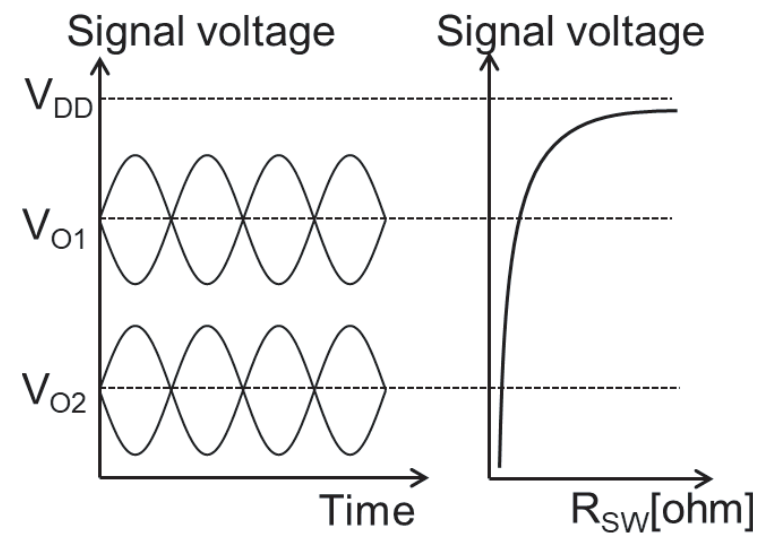

(b)

Fig. 5 Level shift function for facilitating the delay stage. (a) Load circuit of LNA. (b) Bias voltage and on-resistance of NMOS sampling switch.

\section{Stability Analysis}

In this section, the stability of the proposed LNA is described. For differential signal component, the amplifier stage and the driver stage are isolated from each other because node $\mathrm{P}$ serves as AC ground. Therefore, the commonmode stability is addressed here. Figure 4 can be simplified as Fig. 6 for common-mode signal component. The common-mode voltage at the node AP (AN) which is output node of the amplifier stage is determined by a local feedback loop of a CMFB-AMP and M5 (M6). An important point is that the voltage variation at node AP (AN) is not fed back by M3 (M4) because the voltage at node BP (BN) which is output node of the driver stage follows to match the voltage for keeping the drain current of M4 (M3). Variation in the common-mode voltage at the input node is also absorbed by voltage change at node $\mathrm{P}$. Therefore, there is no additional sensitive feedback loop in this current-reuse architecture compared with single-stage differential common-source

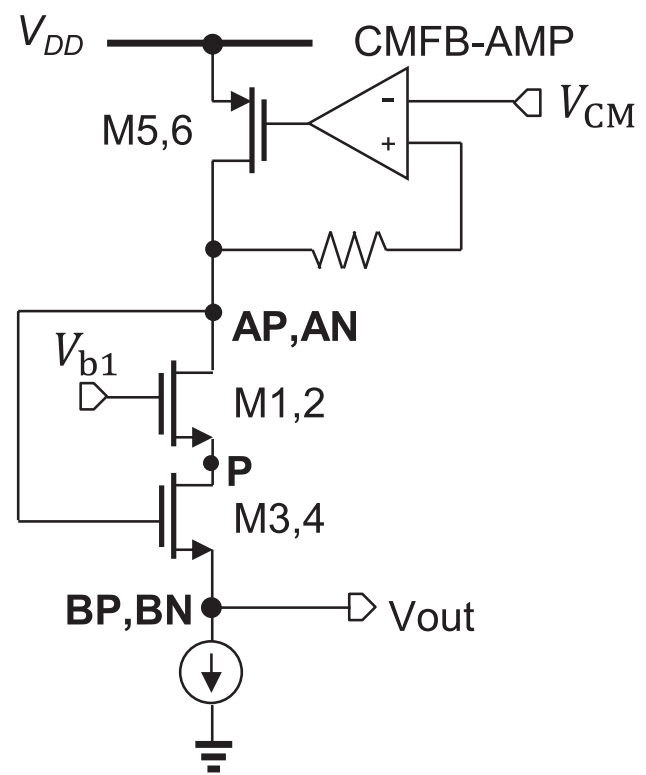

Fig. 6 Simplified schematic for common mode stability analysis.

amplifiers. Therefore, design for stability can be treated in the same manner as an open-loop differential LNA.

\section{Measurement Results}

The proposed LNA was designed and fabricated in a 0.18 $\mu \mathrm{m}$ SOI CMOS process. Figure 7 shows a photo of the fabricated chip. The core area of a single proposed LNA including the CMFB-AMP is $70 \mu \mathrm{m} \times 80 \mu \mathrm{m}$. Figure 8 shows a set up for measuring the fabricated LNA. The fabricated chip is packaged in QFP44. The input signal is supplied to the LNA from arbitrary/function generator (Agilent: 33500B) through the on-chip TR-SW [4]. The output signal of the LNA is buffered by an on-chip monitoring buffer (MON-BUFFER) and measured by a differential probe (Agilent: 1141A) and spectrum analyzer (Keysight: N9030B). In the following measured result, input voltage is defined as voltage at an input terminal on the evaluation board. The reason of using MON-BUFFER is to avoid a parasitic capacitance of evaluation board. Because the LNA is designed to drive a switched capacitor in the delay stage, which is usually a few $\mathrm{pF}$ or less, the parasitic capacitance of evaluation line is too large to evaluate a performance of the LNA without an on-chip buffer. Figure 9 shows a structure of the MON-BUFFER. The MONBUFFER is a very simple source-follower circuit composed of 5V-MOSFETs. 5V-MOSFETs are utilized to prevent a linearity of MON-BUFFER from affecting the evaluation results. The equivalent input capacitance of MON-BUFFER is $1.2 \mathrm{pF}$ and $-3 \mathrm{~dB}$ cutoff frequency with $20 \mathrm{pF}$ load capacitance is $70 \mathrm{MHz}$, which is enough bandwidth to evaluate the LNA. The gain of MON-BUFFER is $-0.07 \mathrm{~dB}$ to $-0.2 \mathrm{~dB}$ in $0.1 \mathrm{MHz}-10 \mathrm{MHz}$, which affects the evaluation of the LNA performance only slightly.

The measured supply current of the LNA circuit was 


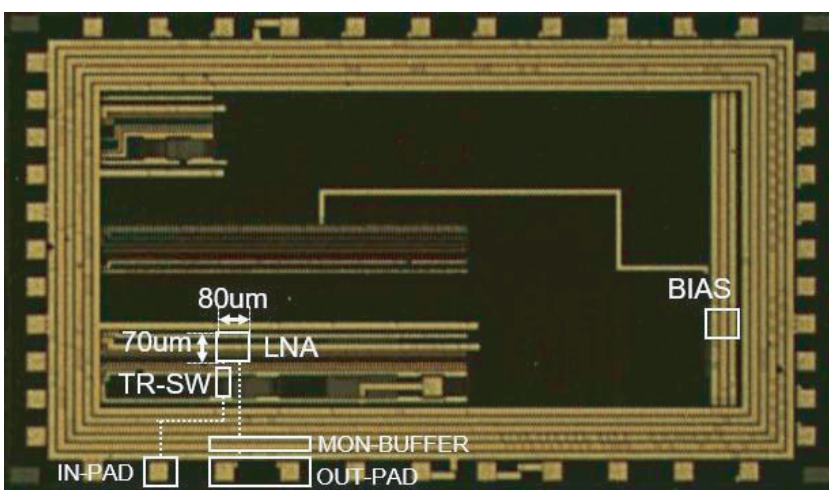

Fig. 7 Die photo of proposed LNA.

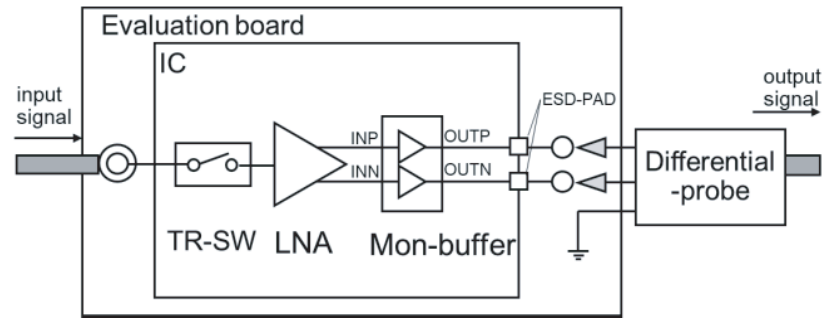

Fig. 8 Measurement setup.

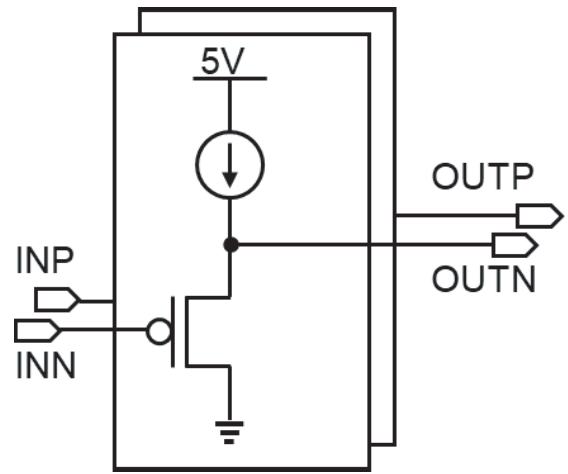

Fig.9 Implemented MON-buffer for measurement.

$85 \mu \mathrm{A}$ including CMFB-AMP under a supply voltage of $1.8 \mathrm{~V}$. The total power consumption was $153 \mu \mathrm{W}$, which is very suitable for ultrasound imaging systems as described in Sect. 1. Figure 10 shows the simulated and measured frequency response of the prototype LNA. The LNA achieved a $10-\mathrm{MHz}$ cutoff at $-3 \mathrm{~dB}$ from the peak gain of $21.9 \mathrm{~dB}$ in measurement results, which is well matched with simulation. In a real ultrasound beamformer, the signal source is the transducer element and their impedance is different from that of signal generator $(50 \mathrm{ohm})$. Regarding the frequency characteristics, the low-frequency side is mainly affected by this difference of impedance. However, since the input impedance of this LNA is high, it can be adjusted to match the impedance of the transducer element by inserting a resistor between the LNA input and the ground. The simulated and measured gain, 2nd and 3rd harmonic distortions versus input voltage swing are presented in Fig. 11. As men-

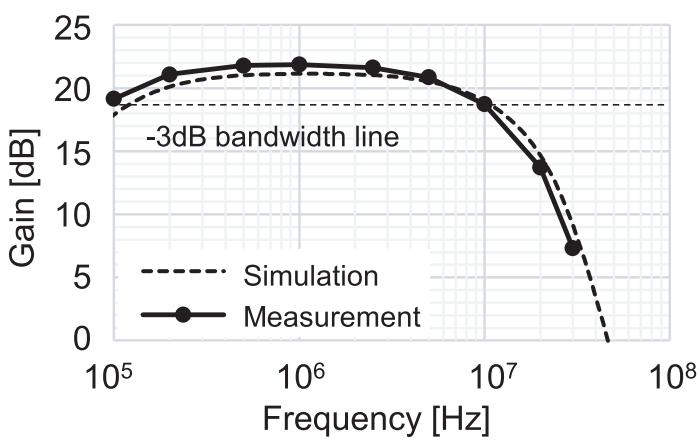

Fig. 10 Simulated and measured frequency response of proposed LNA.

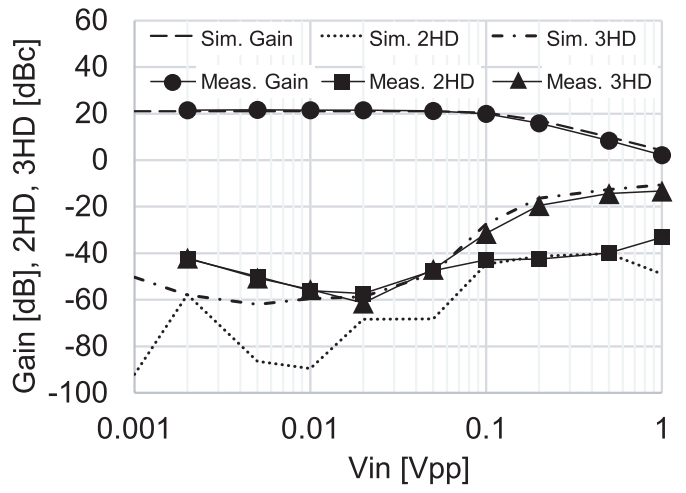

Fig. 11 Simulated and measured performance of gain and 2nd, 3rd harmonic distortion versus input voltage swing.

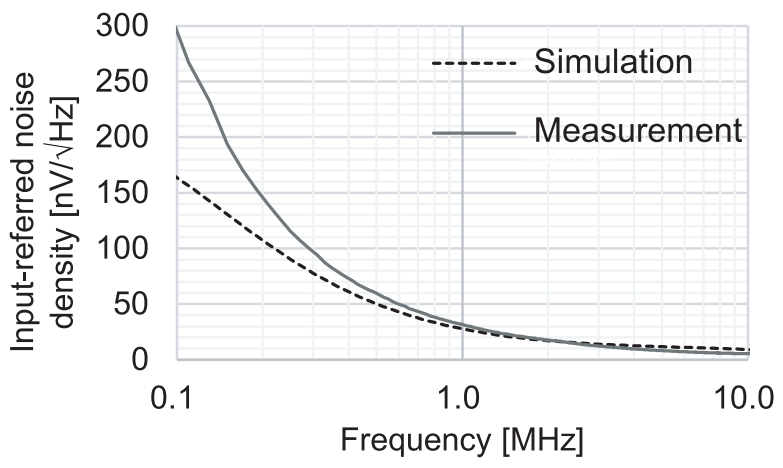

Fig. 12 Simulated and measured input-referred voltage noise density.

tioned in Sect. 2, distortion increases when the input voltage becomes close to $100 \mathrm{mVpp}$, but the 2HD increases less than the 3HD because of the differential cancellation. The 2HD was kept below $-40 \mathrm{dBc}$ at a $0.1-\mathrm{Vpp}$ input in measurement result. A possible reason why the measured $2 \mathrm{HD}$ is higher than the simulation result at small input swing is mismatch in the differential architecture due to process variation. The measured input-referred noise density was $11.3 \mathrm{nV} / \sqrt{ } \mathrm{Hz}$ in average from $1 \mathrm{MHz}$ to $10 \mathrm{MHz}$ (necessary for the ultrasound system), and the noise density of the full bandwidth is plotted in Fig. 12. The performance of the proposed LNA is compared with state-of-the-art works of bandwidth $1 \mathrm{MHz}-$ $100 \mathrm{MHz}$ LNA in Table 1. A noise efficiency factor (NEF) [14] is employed to compare the trade-off between power 
Table 1 Benchmark.

\begin{tabular}{|c|c|c|c|c|}
\hline Work & [11] & {$[12]$} & [13] & $\begin{array}{l}\text { This } \\
\text { work }\end{array}$ \\
\hline Process & $0.18 \mu \mathrm{m}$ & $28 \mathrm{~nm}$ & $65 \mathrm{~nm}$ & $\begin{array}{l}0.18 \mu \mathrm{m} \\
\text { HV SOI }\end{array}$ \\
\hline Supply voltage [V] & 1.8 & 1.0 & 0.5 & 1.8 \\
\hline Gain $[\mathrm{dB}]$ & 19 & 20 & 26 & 21 \\
\hline Bandwidth [MHz] & 33 & 100 & 40 & 9 \\
\hline $\begin{array}{l}\text { Input-referred voltage } \\
\text { noise density }[\mathrm{nV} / \sqrt{\mathrm{Hz}}]\end{array}$ & 1.01 & 1.74 & 7.0 & 11.3 \\
\hline $\begin{array}{l}\text { Total input-referred } \\
\text { voltage noise }[\mu \mathrm{V}]\end{array}$ & 5.8 & 20.8 & 44.3 & 34.0 \\
\hline $\begin{array}{l}\text { 2nd harmonic distortion } \\
{[\mathrm{dBc}]}\end{array}$ & -53.5 & - & -55 & -40 \\
\hline Supply current [mA] & 9 & 2 & 0.108 & 0.085 \\
\hline Noise efficiency factor & 3.7 & 3.6 & 2.8 & 4.0 \\
\hline Core area $\left[\mathrm{mm}^{2}\right]$ & 0.36 & 0.0012 & 0.016 & 0.0056 \\
\hline
\end{tabular}

consumption and noise performance.

$$
N E F=V_{\mathrm{ni}, \mathrm{rms}} \sqrt{\frac{2 I_{\mathrm{tot}}}{\pi \cdot \mathrm{U}_{\mathrm{T}} \cdot 4 \mathrm{kT} \cdot B W}}
$$

where $\mathrm{V}_{\text {ni,rms }}$ is the total in-band input-referred voltage noise, $\mathrm{I}_{\text {tot }}$ is the supply current, $U_{T}$ is the thermal voltage, $\mathrm{k}$ is the Boltzmann constant, $\mathrm{T}$ is the temperature and $\mathrm{BW}$ is the bandwidth of LNA, respectively. The attained power and area efficiencies are competitive or even better than prior works with much finer CMOS process.

\section{Conclusion}

In this paper, a low-power small-size LNA with a novel current-reuse architecture was presented for ultrasound beamformers. The proposed LNA was implemented in only $0.0056 \mathrm{~mm}^{2}$ chip area with $0.18-\mu \mathrm{m}$ CMOS process and verified the performance of an input noise density of $11.3 \mathrm{nV} / \sqrt{ } \mathrm{Hz}$, a bandwidth of $9 \mathrm{MHz}$, and a gain of $21 \mathrm{~dB}$ with sufficiently low supply current $85 \mu \mathrm{A}$, which enables to increase a performance of a next-generation 3D ultrasound imaging system.

\section{References}

[1] Y. Igarashi, S. Kajiyama, Y. Katsube, T. Nishimoto, T. Nakagawa, Y. Okuma, Y. Nakamura, T. Terada, T. Yamawaki, T. Yazaki, Y. Hayashi, K. Amino, T. Kaneko, and H. Tanaka, "Single-chip 3072-element-channel transceiver/128-subarray-channel 2-D array IC with analog RX and all-digital TX beamformer for echocardiography," IEEE J. Solid-State Circuits., vol.54, no.9, pp.2555-2567, Sept. 2019.

[2] J.-Y. Um, E.-W. Song, Y.-J. Kim, S.-E. Cho, M.-K. Chae, J. Song, B. Kim, S. Lee, J. Bang, Y. Kim, K. Cho, B. Kim, J.-Y. Sim, and H.J. Park, "An analog-digital-hybrid single-chip RX beamformer with non-uniform sampling for 2D-CMUT ultrasound imaging to achieve wide dynamic range of delay and small chip area," IEEE ISSCC Dig. Tech. Papers, pp.426-427, Feb. 2014.

[3] K. Chen, H. Lee, and C.G. Sodini, "A column-row-parallel ASIC architecture for 3-D portable medical ultrasonic imaging," IEEE J.
Solid-State Circuits, vol.51, no.3, pp.738-751, March 2016.

[4] S. Kajiyama, Y. Igarashi, T. Yazaki, Y. Katsube, T. Nishimoto, T. Nakagawa, Y. Nakamura, Y. Hayashi, and T. Yamawaki, "T/Rswitch composed of 3 high-voltage MOSFETs with $12.1 \mu \mathrm{W}$ consumption that can perform per-channel TX to RX self-loopback AC tests for 3D ultrasound imaging with 3072-channel transceiver,' IEEE Asian Solid-State Circuits Conference Proceedings of Technical Papers, pp.305-308, Nov. 2019.

[5] J.J. Zhou and D.J. Allstot, "Monolithic transformers and their application in a differential CMOS RF low-noise amplifier," IEEE J. Solid-State Circuits., vol.33, no.12, pp.2020-2027, Dec. 1998.

[6] Z. Huang, C. Huang, Y. Hung, and M. Chen, "A CMOS current reused low-noise amplifier for ultra-wideband wireless receiver," 2008 International Conference on Microwave and Millimeter Wave Technology, pp.1499-1502, Nanjing, 2008.

[7] H. Hsieh and L. Lu, "Design of ultra-low-voltage RF frontends with complementary current-reused architectures," IEEE Trans. Microw. Theory Techn., vol.55, no.7, pp.1445-1458, July 2007.

[8] HA.N. Karanicolas, "A 2.7-V 900-MHz CMOS LNA and mixer," IEEE J. Solid-State Circuits, vol.31, no.12, pp.1939-1944, Dec. 1996.

[9] B. Johnson and A. Molnar, "An orthogonal current-reuse amplifier for multi-channel sensing," IEEE J. Solid-State Circuits, vol.48, no.6, pp.1487-1496, June 2013.

[10] M. Rezaei, E. Maghsoudloo, C. Bories, Y. De Koninck, and B. Gosselin, "A low-power current-reuse analog front-end for highdensity neural recording implants," IEEE Trans. Biomed. Circuits Syst., vol.12, no.2, pp.271-280, April 2018.

[11] S. Jung, S. Hong, and O. Kwon, "Low-power low-noise amplifier using attenuation-adaptive noise control for ultrasound imaging systems," IEEE Trans. Biomed. Circuits Syst., vol.11, no.1, pp.108116, Feb. 2017.

[12] L. Luo, Y. Wu, J. Diao, F. Ye, and J. Ren, "Low power low noise amplifier with DC offset correction at $1 \mathrm{~V}$ supply voltage for ultrasound imaging systems," 2018 IEEE 61st MWSCAS, Windsor, ON, Canada, pp.137-140, 2018.

[13] P. Wang and T. Ytterdal, "A 54- $\mu \mathrm{W}$ inverter-based low-noise singleended to differential VGA for second harmonic ultrasound probes in 65-nm CMOS," IEEE Trans. Circuits Syst. II, Exp. Briefs, vol.63, no.7, pp.623-627, July 2016.

[14] M.S.J. Steyaert and W.M.C. Sansen, "A micropower low-noise monolithic instrumentation amplifier for medical purposes," IEEE J. Solid-State Circuits, vol.22, no.6, pp.1163-1168, Dec. 1987.

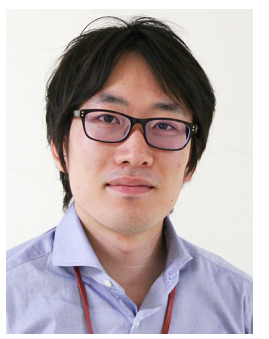

Yohei Nakamura received the B.E. and M.E. degrees in electrical engineering from The University of Tokyo, Tokyo, Japan, in 2009 and 2011, respectively. In 2011, he joined the Central Research Laboratory, Hitachi, Ltd., Tokyo, where he has been engaged in analog circuit design, including ADCs. Mr. Nakamura received the 2014 Young Researcher Award from the Institute of Electronics, Information and Communication Engineers (IEICE). 


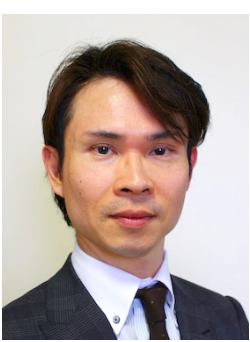

Shinya Kajiyama received the B.E. degree from Yokohama National University, Yokohama, Japan, in 1995, and the M.E. degree in applied chemistry from The University of Tokyo, Tokyo, Japan, in 1997. In 1997, he joined Hitachi, Ltd., Yokohama, where he was engaged in the research and development of readchannel and preamplifier integrated circuits (ICs) for hard disk drives (HDDs), transceiver ICs for high-speed optical links, high-density flash memories, embedded flash memories, microcontroller clocking architecture, and medical ultrasound 2-D array beamformer ICs. Mr. Kajiyama has been serving as a Technical Program Committee Member of the Custom Integrated Circuits Conference (CICC).

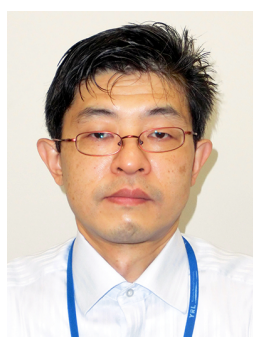

Yutaka Igarashi received the B.S. and M.E. degrees in electronic engineering from the Tokyo University of Science, Chiba, Japan, in 1991 and 1993, respectively. In 1993, he joined the Image and System Laboratory, Hitachi, Ltd., Yokohama, Japan, where he was dedicated to the research and development of high-frequency circuits and RFICs for digital television and cellular phones. Since 2013, he has been engaged in the development of ultrasound 2-D array integrated circuits (ICs) at the Yokohama Research

Laboratory, Hitachi, Ltd.

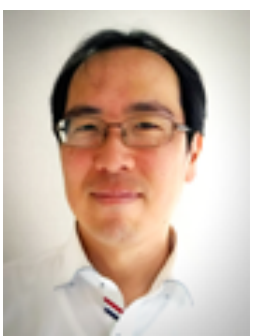

Takashi Oshima received the B.S., M.S., and Ph.D. degrees in physics from The University of Tokyo, Tokyo, Japan, in 1996, 1998, and 2001, respectively. He joined Central Research Laboratory, Hitachi, Ltd., Tokyo, in 2001, where he is currently a Researcher of analog and digital circuits. From 2005 to 2006, he was a Visiting Researcher with the University of California at Berkeley, Berkeley, CA, USA. Dr. Oshima is a member of the Institute of Electronics, Information, and Communication Engineers (IEICE), the Institute of Electrical Engineers of Japan (IEEJ), IEEE and the Physical Society of Japan. He received several awards including the 2010 Best Invited Paper Award of the IEICE Electronics Society and the ISSCC2016 Outstanding Evening Session Award. He served as the Secretary of the IEEE Solid-State Circuits Society Japan Chapter and a Technical Program Committee Member of the IEEE European Solid-State Circuits Conference (ESSCIRC). He is currently a Technical Program Committee Member of the IEEE International Solid-State Circuits Conference (ISSCC).

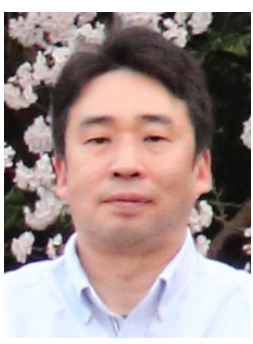

Taizo Yamawaki received the B.S. and M.S. degrees in electronic engineering from Kyoto University, Kyoto, Japan, in 1992 and 1994, respectively. After graduating, he joined the Central Research Laboratory, Hitachi, Ltd., Tokyo, where he developed a series of commercial cellular-phone RFICs for several standards, including GSM, EDGE, WCDMA, and LTE using BiCMOS and CMOS technologies. Since 2013, he has been participating as a Manager in research projects about analog front-end circuits for ultrasound probes, a variety of sensors, and dc-dc converters for automotive applications. 